\title{
Advances in Black Hole Physics and Dark Matter Modelling of the Galactic Halo
}

\author{
Primordial Black Holes and Galactic Formation
}

\author{
Andrew Worsley \\ The WWK Trust, c/o Challis and Associates, Brewery House \\ High Street, Westerham, Kent, TN16 1RG, UK \\ E-mail: andrew.worsley@kcl.ac.uk
}

$\begin{array}{lr}\text { Received: October 17, } 2011 & \text { Accepted: November 2, } 2011 \quad \text { Published: February 1, } 2012 \\ \text { doi:10.5539/apr.v4n1p128 } & \text { URL: http://dx.doi.org/10.5539/apr.v4n1p128 }\end{array}$

\begin{abstract}
One of the most important unresolved issues of modern physics is the presence of cold dark matter (CDM). Such dark matter appears to be essential in explaining the observed galactic rotation curves and the missing Cosmological dark matter. Recent attention has focused on galactic halos as an explanation of the galactic rotation curves, and on the possibility that galactic halos may consist of massive compact halo objects (MACHOs). Indeed results of experiment by the MACHO collaboration group have confirmed the presence of MACHOs in the galactic halo at statistically significant levels. What remains unclear is, what percentage of the galactic halo can be explained by such MACHOs, and what these MACHOs are likely to be composed of. Recent research shows the "most plausible" candidates for such MACHOs are being identified as primordial black holes. Such primordial black holes can also explain quasar brightness, spectral variations, and multiply lensed quasar systems. The presence of these primordial black holes in the galactic halo can be further modelled using advances in black hole gravitational physics. Indeed it has previously been shown that advances in black hole physics can be used to accurately model both CDM associated with the supermassive black hole at the centre of the galaxy, and Cosmological cold dark matter. To establish whether the same mechanism applies to primordial black holes in galactic halos, the original data from the MACHO project are reanalyzed using these advances in black hole physics. It is concluded that the majority of the CDM mass of the galactic halo can be accounted for by MACHOs by using a reanalysis of the data, and by applying advances in black hole gravitational physics. Importantly these advances in black hole physics offer further readily testable gravitational predictions.
\end{abstract}

Keywords: Primordial black hole, Gravitation, Black hole physics, Galactic halo, Cold dark matter, Cosmology 1. Introduction

The presence of CDM remains to date one of the most important unsolved mysteries of modern Cosmology. It is particularly important not only to the understanding of the large scale structure of the Universe, but also in explaining the formation of galaxies. To date the search has concentrated on two main forms of CDM; weakly interactive massive particles (WIMPs), and massive compact halo objects (MACHOs) (Cline 2003). While the presence of WIMPs or some other form of exotic particle is still the favoured model, there has been a recent increase in the interest in MACHOs. Indeed research indicates that the statistical likely hood of MACHOs being present in the galactic halo is high (Alcock et al. 2000a).

The question however, remains as to how prevalent these MACHOs are in the galactic halo, and what form do these MACHOs take? Interestingly, a recent comprehensive review suggests that the most "plausible" form these MACHO's take is that of primordial black holes (Hawkins 2011). In that review, a wide range of experimental evidence from quasar brightness, spectral variations, and multiply lensed quasar systems, and the MACHO project itself, support the presence of primordial black holes in galactic halos (Hawkins 2011). Indeed, results from the MACHO project indicate that the mass of halo objects predominantly range form $0.5-1$ solar masses (Alcock et al. 2000a). This would of course exclude ordinary black holes, whose mass ranges are above 3 solar masses. However, this is exactly the mass range at which primordial black holes are predicted to have arisen (Carr \& Hawking 1974). 
As regards the prevalence of these MACHO's, the results MACHO project were extremely difficult to interpret at the time. Little was known about the physics relating to the galactic halo and numerous models were used to interpret the data. The data were therefore inconclusive, and the standard model used (model S), without knowing what MACHOs were likely to be, suggested that approximately $20 \%$ of the mass of the galactic halo was made of MACHOs. In other models the percentage varied, and in one model (model F) the percentage rose to $40-60 \%$ (Alcock et. al. 2000a).

Following extensive research it has now been deduced that the most likely candidates for these MACHOs are primordial black holes (Hawkins 2011). This allows an updated interpretation of the data. Moreover, the presence of these primordial black holes in the galactic halo can be further modelled using advances in black hole gravitational physics (Worsley 2008). Indeed it has previously been shown that the generic gravitational formula can be used to construct a standard table (Table 1), to explain a dynamic increase in gravity around a black hole. These increases in gravity have already been used to explain the apparent presence of CDM associated with the supermassive black hole at the centre of the galaxy (Worsley 2011a).

Importantly, these advances in black hole physics, have also previously been shown to technically agree exactly with gravitational results at medium and low mass densities (see worked examples Appendix A) (Worsley 2008). They have the advantage of being able to readily predict gravitational time dilation data with precise accuracy, particularly using the data from GPS satellites (see Appendix B). They also technically agree exactly with binary pulsar data (Worsley 2009). Perhaps the most important advantage is that they do not produce infinite density singularities at the centre of black holes.

To establish whether these advanced black hole gravitational mechanisms apply to primordial black holes and can help explain the presence and characteristics of galactic halos, the original data from the MACHO project are used (Alcock et al. 2000a). Here this data from the MACHO project are reanalyzed in the light of these new findings, and with the use of the advances in black hole gravitational physics. Importantly the findings from this study can be confirmed by using additional readily conducted experiments to test the predictions of advanced black hole physics.

\section{Mathematical Methods}

The original published data from the MACHO project are used to reanalyze the presence of MACHOs in the galactic halo (Alcock et al. 2000a). All mathematical calculations follow strict standard algebraic and standard mathematical rules. The gravitational equations used [Eqs. (5 \& 7)], are those of the previously published adaptation to gravity as detailed in the introduction (Worsley 2008, 2011a).

The principle physics proofs are based upon standard physical formulae. The gravitational equations used, offer a high degree of agreement with currently known values compared to GTR. The paper details previously published worked examples which compare advanced gravitational physics to full general relativity (see Appendix A). They have also the advantage of being able to predict gravitational time dilation data with precise accuracy, particularly using the data from GPS satellites (see Appendix B). These technically give exactly the same answers as experiment in low and medium mass density objects, where gravitation has been widely tested. They also technically agree exactly with binary pulsar data (Worsley 2009). The paper also proposes observational experimental methods for the experimental verification of the dynamic increases in gravitation using observations of black holes, as listed in the results and conclusions (see Table 1).

\section{Results}

\subsection{Optical depth and detection efficiency}

This paper analyzes the theoretical and experimental data for the presence of CDM in the galactic halo. In the light of new findings of the likely presence of primordial black holes in the galactic halo (Hawkins 2011), and advances in black hole physics (Worsley 2008, 2009, 2011a), a re-examination of this issue is both timely and important. The data from the MACHO project (Alcock et al. 2000a), will principally be used and reanalyzed in the context of the advances in knowledge of primordial black holes and black hole physics.

The modelling of CDM in the galactic halo is complex, not least as there is little observational data to deduce results from. Beginning with the halo core radius, this is currently estimated at $5 \mathrm{kpc}$. However, the principal limiting observation is the distance to the LMC at $40 \mathrm{kpc}$. This would readily allow the halo core radius to be as large as $25 \mathrm{kpc}$, as in model $\mathrm{F}$ of the MACHO project. Model F predicted that as much as $40-60 \%$ of the halo mass could be accounted for by MACHOs, which already implies that a substantive portion of the halo could be primordial black holes. However, other models showed a lower percentage $20 \%$ of the halo mass due the halo core radius chosen $(5 \mathrm{kpc})$. There were of course other confounding factors. The number of binary and exotic 
lensing events was not directly accounted for in the models. Additionally, the galactic halo of the LMC was unknown, certainly small spiral galaxies are thought to have relatively large halos, and this also needed to be modelled. Thus differing models needed to allow for potential contributions from the LMC halo. However, the results of the MACHO project did reveal that the total contribution of the LMC to the optical depth parameter was in each case very small. Moreover, what has become clear from the MACHO project results, is that one result that was largely independent of the galactic model used was the optical depth. To reduce any modelling complexities the principal parameter used here, will therefore be the optical depth.

The optical depth $(\tau)$ is given by the equation:

$$
\tau=\frac{1}{E} \frac{\pi}{4} \sum_{i} \frac{\hat{t}_{i}}{\varepsilon\left(\hat{t}_{i}\right)}
$$

where $E$ is is the total exposure in object years $\left(6.12 \times 10^{7}\right), \hat{t}_{i}$ is the Einstein ring diameter crossing time of the $i$ th event, and $\varepsilon\left(\hat{t}_{i}\right)$ is the detection efficiency.

The Einstein ring diameter crossing time can itself be derived from the Einstein radius $\theta_{c}$ of a gravitational lens given by the equation:

$$
\theta_{c}=\left(\frac{4 G M}{c^{2} R}\right)^{1 / 2}
$$

where $G$ is the gravitational constant, $c$ is the speed of light, $M$ the mass of the lensing object and $\mathrm{R}$ depends on the angular diameter distances between observer, source and lens (Hawkins 2011).

It is clear from Eq. (2), that the optical depth will be largely model independent, as the two principle terms, the total exposure in object years $(E)$ and the Einstein ring diameter crossing time $\left(\hat{t}_{i}\right)$ are both experimentally measured parameters. This means that the principal estimated value is the detection efficiency $\varepsilon\left(\hat{t}_{i}\right)$.

By using the optical depth, it is therefore the detection efficiency which needs to be carefully examined. In the 2000 paper of the MACHO project, microlensing detection was quite correctly based upon the detection of single lensing objects only. That is because these events can definitively be labelled as microlesning objects (Alcock et al. 2000a). However, the detection efficiency was not adjusted for the possibility that the galactic halo could contain many binary systems. Indeed it is well known that between one half and two thirds of systems in the Milky Way are binary systems, and this figure rises in dense globular clusters. So in the dense regions of a galactic halo these binary systems would be expected to predominate. Indeed the MACHO data showed 4 binary lensing events in the galactic halo, compared to a total of 13 single lensing events (Alcock et. al. 2000a, b). A lower bound to the number of binary systems in the galactic halo should therefore be taken as one third. Moreover, because each binary system would contain 2 objects, then these binary systems would contribute another $2 / 3$ to the mass of the galactic halo, and in turn to the optical depth. Using the strictest subset (subset A) of the MACHO project, this takes the optical depth from $\tau=1.1 \times 10^{-7}$, to $\tau=1.8 \times 10^{-7}( \pm 0.4)$.

However, microlensing detection was strongly in favour of long timescale caustic crossing events. Close binary systems with non caustic shorter timescale crossings would be selected against. Therefore the actual number of binary systems in the galactic halo is more likely to be one half. And because each binary system contains 2 objects this would contribute twice as much again to the mass and optical depth, taking the optical depth to $\tau=$ $3.3 \times 10^{-7}( \pm 0.6)$. This is a significant shift in optical depth, but is still short of the $4.7 \times 10^{-7}$ for a typical halo consisting entirely of MACHOs. Using model F, this would allow the results to tie in with a galactic halo entirely of MACHOs, but this would not apply to the other typical models. What is required to resolve this further, is a fuller understanding of black hole gravitational physics.

\subsection{Black hole gravitational physics and primordial black holes}

Recent published research on gravitational physics shows that both the dark matter associated with Cosmological dark matter, and CDM associated with the black hole at the centre of the galaxy, can be accurately modelled (Worsley 2008, 2009, 2011a). These models use equations that describe a dynamic form of gravity that applies accurately to both ordinary gravity, and black hole gravitational physics [Eq. (3)]. At low mass densities the increase in gravity is small, but nevertheless allows very accurate results. In particular, this dynamic form of gravity technically gives exactly correct results in low and medium mass density objects, where gravity has been 
widely tested (see Appendix A \& B) (For elliptical orbits the equation is slightly different (see worked examples Appendix A).

These equations also give correct answers, for binary pulsar data (Worsley 2009). But in the case of black hole gravitational physics it has been shown there is a progressively larger dynamic increase in gravity, particularly as the black hole event horizon is approached (see Table 1). At the black hole event horizon itself, the force of gravity increases by a factor of 6.25 . As a result this will produce an apparent increase in mass, particularly in the proximity of the event horizon. This explains the apparent presence of CDM at the centre of galaxies (Worsley 2011a). This has also been used to accurately account for the apparent presence of Cosmological dark matter. This means that at the Cosmological event horizon, the dark matter component of the matter in the observable Universe can be predicted to be 6.25 multiplied by that of baryonic matter. Hence dark matter can be predicted to be exactly $84 \%$, and the baryonic component of matter will be exactly $16 \%$. Thus using the generic form of the equation [Eq. (3)], gives answers which are technically in exact agreement with gravitational observation on the scale of the solar system (see Appendix A \& B), at the event horizon of black holes, and at the Cosmological scale.

For the dynamic Newtonian advanced gravitational (DNAg) acceleration equation $\left(\mathrm{a}_{\mathrm{g}}\right)$

$$
a_{g}=\frac{G M}{R^{2}}\left[1+3 G M / R c^{2}\right]^{2}
$$

where $M$ is the larger mass, $c$ is the speed of light and $G$ the gravitational constant, $R$ is the distance, normally taken as the radius for circular orbits ${ }^{\dagger}$.

At the black hole and Cosmological event horizons the same equation [Eq. (3)] can be used. Thus at the event horizon the gravitational equations translate to an increase in the apparent force of gravity by a factor of 6.25 .

Using the generic equation Eq. 3, gives the acceleration due to gravity at the Schwarzschild Radius $\left(\mathrm{R}_{\mathrm{s}}\right)$

$$
a_{g}=\frac{G M}{R^{2}}\left[1+3 G M / R c^{2}\right]^{2}
$$

and

$$
R_{S}=2 G M / c^{2}
$$

thus

$$
a_{g}=\frac{G M}{R^{2}}[1+3 / 2]^{2}=\quad a_{g}=\frac{G M}{R^{2}} x[6.25]
$$

where $\mathrm{M}$ is the larger mass, $\mathrm{c}$ is the speed of light and $\mathrm{G}$ the gravitational constant, $\mathrm{R}$ is the distance, (normally taken as the radius).

Thus in advanced black hole gravity, the acceleration generated at the event horizon would be the normal acceleration due to Newtonian gravity, multiplied by a constant factor. This factor would be 6.25 that of normal Newtonian gravity. Indeed the equations reveal surprisingly precise values for the presence of CDM. In the instances of Cosmological CDM and CDM at the centre of the galaxy, dark matter is predicted to be exactly 6.25 multiplied by the amount of ordinary matter. These results are very much in line with observational experiments on Cosmological CDM. Taking the best estimate for the amount of baryonic matter, $\Omega_{b}=0.0459$, then the total amount of baryonic and dark matter would be exactly 6.25 times this, at $\Omega_{M}=0.287$, and the remaining dark energy is $\Omega_{\Lambda}=0.713$. In keeping with the latest results from type Ia supernovae (Kowalski et al. 2008).

Using these equations it is now possible to interpret the MACHO project results relating to the primordial black holes in the galactic halo. Given the high density of matter in the galactic halo, a conservative estimate allows for one half of the systems in the galactic halo to be in the form of binary systems (see Results section 3.1). Equally close orbits of these binary systems can be directly deduced from the age of the galaxy and the resulting extensive gravitation radiation damping.

Indeed, the question remains, as to why there appears to have been so little coalescence of these binary systems, to give higher mass MACHOs. In advanced black hole gravitation, this can be explained by the dynamic increase 
in gravity as the black holes approach coalescence. Because of the apparent increase in force, this means the black holes are given additional "boost" in angular momentum. This would in turn prevent coalescence. Certainly black holes are experimentally observed to have slower orbital decays than other high mass binary objects (Bagot 1996). This boosting tendency would increase the closer the binaries came (see Table 1).

Equally, there are no accretion discs to impede motion or tidal torques to cause orbital decay. These binaries would thus have a high degree of synchronism (Bagot 1996). These dynamic mechanisms means the primordial black holes are therefore delayed from coalescence, and would remain in close binary orbits. These gravitational effects will start to become significant at less than 48 Schwarzschild radii, as the gravitational force starts to rise significantly when the radius is less than this (Table 1). In effect that means that these systems will be held in close orbit once the binaries are closer than 48 Schwarzschild radii. This observation explains why the average mass of the black holes seen in the MACHO project, is still that of the original primordial black holes from which they arose. Specifically, as the dynamics of the orbits would tend to prevent coalescence, this mechanism thus explains why these primordial black holes have not seen substantive mass increases due to coalescence.

Moreover, these close binary orbits would have an effect on the optical depth of these systems as the apparent mass of these binary black hole systems would rise by the square of the increase in gravitational mass, as given in Table 1. A number of models can be developed, for instance the incidence of binaries could be greater than one half, or the closeness of the binary systems could be relaxed. However, the preferred galactic halo model, is for the incidence of binaries to be one half of all systems, and for the presence of close binary systems. In close binary systems it is estimated that $1 / 3$ of binary black holes are at distances greater than 48 Schwarzschild radii, $1 / 3$ would be on average at 24 Schwarzschild radii, and 1/3 would be at on average at 12 Schwarzschild radii. Using the strictest data subset (Subset A) from the MACHO project, then the optical depth in this case is, $\tau=$ $4.26 \times 10^{-7}( \pm 0.7)$. These results are within the expected optical depth range of $\tau=4.7 \times 10^{-7}$, for a galactic halo consisting almost entirely of MACHOs.

\section{Discussion}

\subsection{Analysis of the MACHO Project data}

Analysis of the data from the MACHO Project has identified the presence of MACHOs in the galactic halo at highly statistically significant levels (Alcock et al. 2000a). Additionally, the project data did identify the likely mass range of these MACHOs at 0.5-1 solar masses. In the light of this mass range the first advance in the analysis is regards the constituents of the galactic halo. The mass range found excluded ordinary black holes, whose mass is known to be over 3 solar masses. Other stellar mass astronomical objects such as white dwarfs are largely excluded as in the majority of cases they should still be visible. Neutron stars remain a possibility, but these usually also give off some sort of telltale sign in the form of pulsar beams or X-ray emissions. That leaves primordial black holes as the "most plausible" candidates for these MACHOs (Hawkins 2011). The mass ranges of these primordial black holes have been previously predicted using theoretical work (Carr \& Hawking 1974).

The equation for the solar mass horizon $\left(M_{h}\right)$ is given by the equation:

$$
M_{h} \approx \frac{c^{3} t}{G}
$$

where $c$ is the speed of light, $t$ is the time form the Big Bang, and $G$ is the gravitational constant.

In brief, after the Big bang the phase transition at the quark hadron QCD phase, which occurred at $10^{-5} \mathrm{sec}$, was the optimum period for primordial black hole formation. This resulted in the prevalence of primordial black holes at 0.5-1 solar masses. Interestingly, this is exactly the mass range at which the MACHO project found microlensing effects towards the Large Megallenic Cloud (LMC). Indeed a reanalysis of the data including the estimated number of binary MACHO's (Alcock 2000b), suggests that the optical depth of these primordial black holes could be greater than first reported (Alcock 2000a). Thus it is possible that these primordial black holes, could account for a greater degree of the optical depth required to explain the galactic rotation curves.

Qualitative predictions are also very useful. It is possible to use the presence of these primordial black holes to predict how and why galaxies began to form so early after the Big Bang. Galaxies have now been seen forming as far back in time as just 480 million years after the Big Bang. This is far too fast for a straight forward bottom up approach. But the formation of supermassive black holes at the centre and the formation of halos around the galaxy from primordial black holes can explain this rapid formation. Indeed the presence of primordial black 
holes in the Big Bang would explain galactic formation very well. The larger primordial black holes probably coalesced at the centre of the galaxy whilst the smaller ones were ejected to form the galactic halo.

A galactic halo with primordial black holes also explains why galaxies such as the pinwheel galaxy are so perfectly formed, even without a large central black hole. The mergers of spiral galaxies to form elliptical galaxies can also be explained. Moreover, one can readily predict that these merged ellipticals will have a large central black hole, and will be strongly gravitationally lensing objects. But on the other hand the galactic halos of the elliptical galaxies will be depleted by successive collisions. This explains why elliptical galaxies have not been shown to have significant galactic halos (Cline 2003). Qualitatively it is also possible to explain the bullet cluster quite directly, as the two different populations of black holes are segregated. The supermassive black hole and the associated visible matter are in one part, which can be seen to result in gravitational lensing. The dark matter halo drags out the gas and heats it, which can be seen by the X-ray telescope CHANDRA in a separate bullet shaped shock wave. It is difficult to model these results with ordinary modified Newtonian Dynamics (MOND) alone (Pointecouteau \& Silk 2005). To model this requires both MACHOs, predominately in the form of solar mass primordial black holes in the galactic halo, along with dynamic advances in black hole gravitational physics.

What is interesting about the bullet cluster is that the majority of the gas appears to have followed the dark matter halo, which implies that the total mass of this halo is greater than the visible mass. Thus the primordial black holes in these galactic halos can also contribute to solving the missing baryon problem (Bregman 2007). It is thus possible to estimate the break down of the constituents of the Universe into baryonic matter from stars $(\mathrm{sb})$, baryonic matter from primordial or other black holes (bb), and baryonic matter from gas (gb). Thus we have $\Omega_{s b}=0.004, \Omega_{b b}=0.0119, \Omega_{g b}=0.030$. This agrees well with the best estimate of the total baryon mass, $\Omega_{b}=0.0459$. According to the advanced gravitational physics presented here, then the total amount of baryonic and dark matter would be exactly 6.25 times that of the total baryon mass. Thus the total amount of baryonic and dark matter is $\Omega_{M}=0.287$, and the remaining dark energy is $\Omega_{\Lambda}=0.713$. These results are in very good agreement with the latest results from type Ia supernovae (Kowalski 2008).

\subsection{Advanced black hole gravitational physics}

To fully explain the galactic rotation curves further, advances in black hole gravitational physics are required. Such an advanced gravitational equation [Eq. (3)] has been previously developed (Worsley 2008, 2009, 2011a). This equation can also readily be applied to primordial black hole gravitational physics. This generic gravitational formula predicts a dynamic increase in the force of gravity, which has been shown to give very accurate results for low and medium density objects. The predicted results are technically in exact keeping with the results of gravitational experiments, where gravitation has been widely tested (see Appendix A) They have also the advantage of being able to predict gravitational time dilation data with extreme accuracy (to one part in $10^{11}$ ), particularly using the data from GPS satellites (see Appendix B). They also technically agree exactly with binary pulsar data (Worsley 2009).

Using Eq. (3), there is a significant increase in gravity in the proximity of a black hole. Table 1 shows the dynamic increase in gravity as the Schwarzschild black hole event horizon is approached. At 12 Schwarzschild radii the acceleration due to gravity, rises by a factor of 1.2656, compared to Newtonian gravity. At 3 Schwarzschild radii the acceleration due to gravity rises by a factor of 2.25. At 1.5 Schwarzschild radii the acceleration due to gravity rises by a factor of 4 . In particular, at the black hole event horizon itself, the force of gravity increases by a factor of 6.25 . As a result this will produce an apparent increase in mass, particularly in the proximity of the event horizon. This has also been used to accurately account for the apparent presence of Cosmological dark matter. This means that at the Cosmological event horizon, the dark matter component of the matter in the observable Universe can be predicted to be exactly $84 \%$, and the baryonic component of matter will be exactly $16 \%$.

However, direct observation of black hole physics has not previously been tested. Importantly, accurate predictions of black hole physics have been made (Worsley 2011), which are readily testable, using established methods (Schodel et al. 2002). The advanced black hole physics presented here, also has the advantage that it obviates the need for the formation of infinitely dense singularities. Indeed ordinary black holes are predicted to be very dense, and increasingly dense with increasing mass. These very high levels of density also mean that black holes, can sustain very close orbits without suffering orbital decay due to tidal forces (Bagot 1996). This helps to explain the finding that significant coalescence of MACHO objects does not appear to have occurred in the galactic the halo, as close orbit are sustainable (Hawkins 2011). In particular, using advanced gravitational physics the primordial black holes are predicted to be considerably denser than ordinary black holes, but still not 
infinitely dense. Moreover, as there is a residual Planck volume to the black hole mass, advanced black hole gravitation can be translated into quantum format (Worsley 2009). This advanced quantum gravity is formatted in a way that also accounts for the presence of dark energy (Perlmutter et al. 1997, Caldwell 2004) This also allows advanced quantum gravity to dovetail in with quantum physics as a whole (Worsley 2010).

\section{Conclusions}

In this paper the data from the MACHO project are reanalyzed in the light of two recent advances regarding the galactic halo of the Milky Way (MW) galaxy. It is first important to note that the MACHO study did confirm the presence of microlensing objects within the galactic halo, to a high level of statistical significance. Moreover, a recent comprehensive review has concluded that the "most plausible" candidates for these MACHO's are primordial black holes (Hawkins 2011). Indeed, previous predictions of the mass of these primordial black holes (Carr \& Hawking 1974), agrees closely with the mass results of the MACHO project, specifically that the MACHOs are between 0.5- 1 solar masses. Additionally, a number of other recent astronomical observations such as quasar brightness, spectral variations, and multiply lensed quasar systems have identified primordial black holes as the prime candidates for MACHOs (Ashford, McDonald, \& Spergel 2003, Hawkins 2011).

The second recent advance relates to the physics of black holes. It has previously been shown that the modelling of the supermassive black holes at the centre of galaxies can account for the apparent presence of CDM there (Worsley 2011a). This can be accurately modelled using gravitational data from Table 1. Moreover, this modelling allows very precise predictions for astronomical objects that orbit black holes, particularly the supermassive black hole at the centre of the MW galaxy. Indeed it is suggested that experiment, should be used to check these predictions regarding the galactic supermassive black hole, by observing close orbits around the supermassive, or indeed any ordinary black hole (Schodel et al. 2002). The presence of supermassive black holes at the centre of other galaxies and clusters of galaxies and the respective advances in black hole physics also accounts for the observed galactic lensing phenomena (Cline 2003). Equally well using these same advances in black hole gravitational physics Cosmological CDM has previously been modelled and accurately accounted for (Worsley 2008, 2009).

Overall, in this paper it is shown that the presence of primordial black holes can account for the optical depth of the galactic halo. Using a reanalysis of the strictest data subset (Subset A) from the MACHO project, the optical depth in this case is, $\tau=4.26 \times 10^{-7}( \pm 0.7)$. These results are within the expected optical depth range of $\tau=4.7 \times$ $10^{-7}$, for a galactic halo consisting almost entirely of MACHOs. Theses estimates are in close agreement with the predicted optical depth given by the galactic rotation curves (Hawkins 2011).

\section{Acknowledgements}

The assistance of the WWK Trust, a charitable trust whose aim is to support fundamental research in all areas of scientific endeavour, is acknowledged.

\section{References}

Alcock, C. et al. (2000a). The MACHO project: Microlensing Results from 5.7 Years of LMC Observations. Astrophys. J. 542: 281.

Alcock, C. et al. (2000b). Binary microlensing events form the MACHO project. The Astrophys. J. 541: 270.

Ashford, N. McDonald, \& P. Spergel D. N. (2003). Primordial Black Holes as Dark Matter: the Power Spectrum and Evaporation of Early Structures. Asrophys J. 594: L71-L74.

Bagot, P. (1996). Boost of the orbital motion in high mass X-Ray binaries. Astro. and Astrophys. J. 314: 576.

Bregman, J. N. (2007). The search for the Misssing Baryons at Low Redshift. Annu. Rev. Astro. Astrophys., 45: 221.

Caldwell, R. R. (2004). Dark Energy. Phys. World. 17: (3) 1183-1207.

Carr, B. J., \& Hawking S. W. (1974). Black Holes in the early Universe. Mon. Not. Roy, Astron. Soc. 168: 399.

Cline, D. $5^{\text {th }}$ (2003). International Symposium on the Detection of Dark matter and Dark Energy in the Universe. Nuc. Phys.s B, Proceedings Supplement. edited by D. Cline. Elsevier Amsterdam.

Hawkins, M. R. S. (2011). The case for primordial black holes as dark matter, Mon. Not. Roy. Astron. Soc. 415: 2744

Kowalski, M. et al. (2008). Improved Cosmological Constraints from New, Old and Combined Supernova Data Sets. Astrophys. J. 686: 749. 
Perlmutter et al. (1997). Measurements of the cosmological parameters of Omega and Lambda from the first 7 supernovae of $\mathrm{z}>0.35$. Astrophys $J$. 483: 565.

Pointecouteau, E., \& Silk J. (2005). New Constraints on MOND from Galaxy Clusters. Mon. Not. Roy, Astron. Soc. 364: 654-658.

Schodel, R. et al. (2002). A star in a 15.2-year orbit about the super-massive black hole at the centre of the Milky Way. Nature 494: 694.

Worsley, A. (2008). An advanced dynamic adaptation of Newtonian equations of gravity. Phys. Essays, 21: 222-228. http://dx.doi.org/10.4006/1.3027501

Worsley, A. (2009). String quintessence and the formulation of advanced quantum gravity. Phys. Essays, 22: 364-377. http://dx.doi.org/10.4006/1.3182733

Worsley, A. (2010). Harmonic quintessence and the formulation of a fundamental energy equivalence equation. Phys. Essays, 23: 311-319. http://dx.doi.org/10.4006/1.3392799

Worsley, A. (2011). Advances in Black Hole Gravitational Physics and Cold Dark Matter Modelling. Open Astron. J. 4: 6-13. http://www.benthamscience.com/open/toaaj/articles/V004/6TOAAJ.pdf

\section{Appendix A}

\section{Advances in the perihelia of solar system bodies.}

1). Advance in the Perihelion of Mercury (worked example)

$$
\Delta \operatorname{circ}=3 G M s /\left(c^{2} a\left(1-e^{2}\right)\right)=7.987 \times 10^{-8}
$$

multiplied by the No. of orbits in a century,

$$
=\quad 3.316 \times 10^{-5}
$$

the ratio of circumference to arc second

$$
=360 \times 3600=1.296 \times 10^{6}
$$

calculated advance in the perihelion of Mercury per century

$$
3.316 \times 10^{-5} \times 1.296 \times 10^{6}=\underline{42.98 \mathrm{arcsec}} .
$$

Equivalent general relativistic value per century

$$
=42.98 \mathrm{arc} \mathrm{sec}
$$

Experimentally estimated advance in the perihelion of Mercury per century (Ohanian \& Ruffini 1994)

$$
=43 \pm 0.1 \text { arc sec }
$$

where $\Delta_{\text {circ }}$ is the change in circumference of the orbit of Mercury, G is the gravitational constant, Ms the mass of the Sun, $\mathrm{c}$ the speed of light, a is the semi major axis of Mercury 's orbit (in meters), e is the eccentricity.

2). Advance in the Perihelion of Earth (worked example)

$$
\Delta \operatorname{circ}=3 G M s /\left(c^{2} a\left(1-e^{2}\right)\right)=2.961 \times 10^{-8}
$$

in a century

$$
=2.961 \times 10^{-6}
$$

the ratio of circumference to arc second

$$
=360 \times 3600=1.296 \times 10^{6}
$$

calculated advance in the perihelion of Earth per century

$$
2.961 \times 10^{-6} \times 1.296 \times 10^{6}=\underline{3.84 \text { arc sec. }} .
$$

Equivalent general relativistic value per century

$$
=3.84 \text { arc sec }
$$

Experimentally estimated advance in the perihelion of Earth per century (Ohanian \& Ruffini 1994).

$$
=3.84 \pm 0.1 \mathrm{arc} \mathrm{sec}
$$

where $\Delta_{\text {circ }}$ is the change in circumference of the orbit of Earth, G is the gravitational constant, Ms the mass of the Sun, c the speed of light, a is the semi major axis of Earth's orbit (in meters), e is the eccentricity. 
3). Advance in the Perihelion of Mars (worked example)

change in the circumference of Mars orbit:

$$
\Delta \operatorname{circ}=3 G M s /\left(c^{2} a\left(1-e^{2}\right)\right)=2.961 \times 10^{-8}=1.9595 \times 10^{-8}
$$

multiplied by the number of Mars orbits in a century (53.15):

$$
=1.0416 \times 10^{-6}
$$

the ratio of circumference to arc second

$$
360^{\circ} \times 3600=1.296 \times 10^{6}
$$

calculated advance in the perihelion of Mars per century

$$
1.0416 \times 10^{-6} \times 1.296 \times 10^{6}=1.35 \mathrm{arcsec} / \mathrm{cy}
$$

Equivalent general relativistic value per century

$$
=1.35 \mathrm{arcsec} / \mathrm{cy}
$$

Experimentally estimated advance in the perihelion of Mars per century [11].

$$
=1.35 \pm 0.1 \mathrm{arcsec} / \mathrm{cy}
$$

where $\Delta_{\text {circ }}$ is the change in circumference of the orbit of Mars, $\mathrm{G}$ is the gravitational constant, $M_{S}$ is the mass of the Sun, c the speed of light, a is the semi major axis of Mars orbit (in meters), e is the eccentricity.

\section{APPENDIX B}

\section{Calculation of Gravitational Time Dilation}

1). Calculation of Gravitational Time Dilation relative to GPS satellites (worked example)

$$
\begin{gathered}
\Delta t=G M /\left(c^{2} R\right) \\
\Delta t=G M_{E} / c^{2}=4.436 \times 10^{-3}
\end{gathered}
$$

The total radius $R$ is given as the altitude of GPS $=20,184 \mathrm{~km}$, plus the radius of the Earth $R_{E}=6,372 \mathrm{~km}$.

Thus for the GPS satellite $\mathrm{R}=26,556 \mathrm{~km}$ :

$$
\Delta t=G M /\left(c^{2} R\right)=1.670 \times 10^{-10}
$$

For Earth bound obersvers:

$$
\Delta t=G M_{E} /\left(c^{2} R_{\mathrm{E})}=6.959 \times 10^{-10}\right.
$$

The time difference ratio for the satellite is:

$$
6.959 \times 10^{-10}-1.670 \times 10^{-10}=5.289 \times 10^{-10}
$$

Multiplied by the number of seconds in a day 86,400

$$
\Delta_{t}=45.7 \mu \mathrm{sec} / \text { day. }
$$

Observed gravitational time dilation relative to GPS, $\Delta_{\mathrm{t}} \approx 45 \mu \mathrm{sec} /$ day.

where $\Delta_{t}$ is the change in time, $G$ is the gravitational constant, $M_{E}$ is the mass of the Earth, $R_{E}$ is the radius of Earth, $R$ is the total orbital Radius, $c$ the speed of light. 
Table 1. Acceleration due to gravity $\left(\mathrm{a}_{\mathrm{g}}\right)$, in the proximity of a black hole, using dynamic Newtonian advanced gravitation (DNAg)

\begin{tabular}{|ll|}
\hline 1$) . R=48 R_{S}:$ & $a_{g}=\frac{G M}{R^{2}} x[1+1 / 32]^{2}=\frac{G M}{R^{2}} x[1.0635]$ \\
\hline 2). $R=24 R_{S}:$ & $a_{g}=\frac{G M}{R^{2}} x[1+1 / 16]^{2}=\frac{G M}{R^{2}} x[1.1289]$ \\
\hline 3). $R=12 R_{S}:$ & $a_{g}=\frac{G M}{R^{2}} x[1+1 / 8]^{2}=\frac{G M}{R^{2}} x[1.2656]$ \\
\hline 4). $R=6 R_{S}:$ & $a_{g}=\frac{G M}{R^{2}} x[1+1 / 4]^{2}=\frac{G M}{R^{2}} x[1.5625]$ \\
\hline 5). $R=3 R_{S}:$ & $a_{g}=\frac{G M}{R^{2}} x[1+1 / 2]^{2}=\frac{G M}{R^{2}} x[2.25]$ \\
\hline 6). $R=1.5 R_{S}:$ & $a_{g}=\frac{G M}{R^{2}} x[1+1]^{2}$ \\
\hline 7). $R=1 R_{S}: \quad a_{g}=\frac{G M}{R^{2}} x[1+3 / 2]^{2}=\frac{G M}{R^{2}} x[6.25]$ \\
\hline
\end{tabular}

Where $R$ is the radius of the in-falling object from the black hole, $R_{S}$ is the Schwarzschild radius, $G$ is the gravitational constant, $M$ is the mass of the black hole. Answers given to 4 decimal places. 